\title{
Predicting survival and risk stratification based on nomogram for locally advanced cervical cancer patients of the 2018 FIGO staging system treated with radiochemotherapy in southern China: a retrospective study
}

\section{Yong Zhang ( $\nabla$ zhangyonggxmu@163.com )}

First Affiliated Hospital of GuangXi Medical University

Xinshu Li

First Affiliated Hospital of GuangXi Medical University

Lulu Huang

First Affiliated Hospital of GuangXi Medical University

\section{Li Jiang}

First Affiliated Hospital of GuangXi Medical University

\section{Shanshan Ma}

First Affiliated Hospital of GuangXi Medical University

Meizhu Shen

First Affiliated Hospital of GuangXi Medical University

Yanmei Que

First Affiliated Hospital of GuangXi Medical University Jinting Que

First Affiliated Hospital of GuangXi Medical University

Nianqun Qin

First Affiliated Hospital of GuangXi Medical University

\section{Fang Wu}

First Affiliated Hospital of GuangXi Medical University

\section{Research Article}

Keywords: Locally advanced cervical cancer (LACC), prognostic factors, nomogram, 2018 FIGO staging system, radiochemotherapy, Elastic net regression

Posted Date: February 1st, 2022

DOI: https://doi.org/10.21203/rs.3.rs-1309255/v1 
License: (c) (i) This work is licensed under a Creative Commons Attribution 4.0 International License. Read Full License 


\section{Abstract}

Background: Concurrent chemoradiation has been the standard treatment for locally advanced cervical cancer patients worldwide. This study was conducted to construct the nomogram of prognosis and risk stratification for locally advanced cervical cancer in southern China

Material and Methods: The medical records of 258 LACC patients who mostly received cisplatin-based concurrent chemoradiation and high-dose-rate intracavity brachytherapy at the first affiliated hospital of Guangxi Medical University were analyzed. Stepwise selection of 22 factors with the Elastic net method was used to obtain a predictive model and construct nomograms for both progression-free survival (PFS) and overall survival (OS).

Results: The median follow-up period was 47 months. The 3 -year and 5 -year OS were $73.7 \%$ and $65.9 \%$, respectively, while the 3-year and 5-year PFS were $68.1 \%$ and $60.2 \%$. In the multivariable analysis, different cumulative cisplatin dose ( $<180 \mathrm{mg} / \mathrm{m}^{2}$ : HR: $0.099,95 \% \mathrm{Cl}: 0.010-0.909, p=0.040 ; 180-$ 200mg/m²: HR: 0.058, 95\% Cl: 0.006-0.545, $p=0.012 ;>200 \mathrm{mg} / \mathrm{m}^{2}:$ HR: $0.050,95 \% \mathrm{Cl}: 0.006-0.520$, $p=0.012$ ), bulky tumors (HR: $1.869,95 \% \mathrm{Cl}: 1.127-3.098, p=0.015)$, ICBT (HR: $0.385,95 \% \mathrm{Cl}: 0.218-0.681$, $p=0.001)$, and thrombocytopenia pre-treatment (HR: 6.832, 95\% Cl: $1.169-39.902, p=0.032)$ had significant effect on PFS, and OS was influenced by bulky tumors (HR: 1.916, 95\% Cl: 1.100-3.336, $p=0.021$ ), ICBT (HR: 0.499, 95\% Cl: 0.252-0.986, $p=0.045$ ), thrombocytopenia pre-treatment (HR: 3.865 , $95 \% \mathrm{Cl}: 1.367-10.922, p=0.010)$ and high neutrophilic granulocyte percentage level in early stages of the treatment (HR: 2.041, 95\% Cl: 1.172-3.552, $p=0.011)$.Nomograms of the independent predictors were established (cross-validated concordance probability $c=0.671$ for PFS, and 0.673 for OS). Based on the scores of the nomogram, subgroups of risk stratification further constructed showed a statistically difference in survival $(p<0.05)$.

Conclusions: Our study shows the prognostic value of nomogram to predict survival and establish risk stratification for LACC patients from southern China. Future individualized tailoring of therapy should focus even further on the high-risk patient populations.

\section{Introduction}

Among the most common cancers in women, cervical cancer is one of the leading causes of cancerrelated deaths, ranking as the fourth in frequency and mortality worldwide and the second behind breast cancer in developing countries with approximately 604,000 global new cases and 342,000 deaths in $2020^{1}$. In China, owing to the imbalance of regional development, there are about 131,500 new cases and 53,000 deaths, with a crude incidence rate of 15.3 women per 100,000 annually ${ }^{2}$.

Five large prospective randomized clinical trials have revealed that concurrent chemoradiation (CCRT), especially with cisplatin-based chemotherapy, prolongs the disease-free survival while reducing mortality of patients with locally advanced cervical cancer (LACC) $)^{3-7}$. With developments in techniques sharply 
increasing the local control ratio of LACC for decades, different predictive and prognostic factors and reported most in common were as follows: FIGO stage (2009), histology, tumor size, pelvic and/or paraaortic lymph node status, age, delivery of chemotherapy. In 2018, the International Federation of Gynecology and Obstetrics (FIGO) revised its staging system for cervical cancer, taking into account the detrimental effect of regional lymph node metastases and other factors on cervical cancer survival. An important change from the previous staging system is that the new staging system designates patients with regional lymph node metastases as stage IIIC, whose aim was to provide more stratified and accurate prognostic groups.

Nearly ten years, nomograms are universally used as prognostic devices in medicine and oncology in order to help clinical decision and prognostication. Several nomograms of prognostic factors for LACC patients in developed regions of the world have been created over the years ${ }^{8}$, However, there are few nomograms related to LACC patients of the 2018 FIGO staging system in less-developed areas recently.

In China, despite the significant improvements in screening techniques, diagnostic procedures, and treatment modalities to decrease the incidence and mortality of LACC, the mortality rate remains at a high level, and distant disease control, as well as overall survival, is still a concern, especially in comparatively less-developed southern China. With the high infection rate of HPV, the lack of effective screening and vaccination, a large number of patients in these areas presented at a relatively advanced stage with highrisk factors such as large tumor size and regional lymph node metastasis when first diagnosed. In face of the increasing new cases, unsatisfactory survival, and the lack of research on the correlated crowd, this current study was conducted to construct the predictive model of prognosis and further risk stratification through nomogram for LACC patients treated in a general hospital in southern China, hoping to help trigger more individualized and adequate treatment decisions in the future.

\section{Methods}

\section{Patients}

A total number of 258 patients with locally advanced cervical cancer treated in the first affiliated hospital of Guangxi Medical University between January 2016 and February 2020 were enrolled in this retrospective study. The inclusion criteria were as follows: (a) pathologically proven uterine cervical cancer with histological subtypes of squamous cell carcinoma (SCC), adenocarcinoma, adenosquamous cell carcinoma, and other types; (b) identified LACC (including $\triangle A 1$ to $\triangle A$ ) according to the latest 2018 guidelines of the Federation International of Gynecology and Obstetrics (FIGO); (c) no definite evidence of distant organ metastases observed on imaging. The exclusion criteria were as follows: (a)combing with other cancers; (b) severe concomitant illness such as uncontrolled cardiovascular disease; (c) receiving chemotherapy, radiotherapy, or pelvic surgery pre-treatment.

Each patient was required a gynecological examination to subjectively estimate the clinical size of the tumor via visual and palpation. In addition to a pathological biopsy, Magnetic resonance imaging (MRI) 
and/or computed tomography (CT) were performed to help to detect the size of the primary lesion, and the bulky tumor was defined as those whose maximum diameter on imaging is greater than or equal to $4 \mathrm{~cm}$. Under additional approval by the patient, the positron emission tomography/computed tomography (PET/CT) was applied after the previous MRI/CT examination as a supplement. For patients who received $\mathrm{PET} / \mathrm{CT}$, pelvic and para-aortic lymph nodes with FDG accumulation greater than the liver accumulation of standard uptake value (SUV) $>2.5 \mathrm{ng} / \mathrm{ml}$ were diagnosed as lymph nodes metastasis. For those without PET/CT, lymph nodes with a short-axis diameter longer than $1 \mathrm{~cm}$ were defined as positive lymph nodes. Once diagnosed, each patient's standard treatment was explained, followed by approval through written informed consent. We obtained other clinicopathological data such as age at diagnosis, the chief complaint from the electronic medical record.

\section{Treatment}

Enrolled patients received pelvic external beam radiotherapy (EBRT) with three-dimensional conformal radiotherapy (3DCRT) or intensity-modulated radiotherapy (IMRT) using fractions of 1.8 to 2 Gy, which were delivered up to five times a week for a total dose of 45 to $50 \mathrm{~Gy}$. Meanwhile, a part of patients irradiated by IMRT received simultaneous integrated boost (SIB), in which positive or suspicious positive lymph nodes were defined as gross tumor volume (GTVnd) with a higher dose of 56-60Gy. During the late treatment period, High-dose-rate intracavity brachytherapy (ICBT) delivering a total dose of 30-36 Gy in 5-6 fractions to point A was performed 2-3 times per week for most patients. Most patients were given according to one of the three following cisplatin-based regimens: (a) cisplatin ( $\left.40 \mathrm{mg} / \mathrm{m}^{2}\right)$ at weekly intervals for a total of $5-6$ cycles.; (b) cisplatin $\left(60 \mathrm{mg} / \mathrm{m}^{2}\right.$ on day 1$)$ and $5-\mathrm{FU}\left(1000 \mathrm{mg} / \mathrm{m}^{2}\right.$ per day on days $1-4)$ at tri-weekly intervals for a total of $2-3$ cycles; (c) cisplatin $\left(60-80 \mathrm{mg} / \mathrm{m}^{2}\right)$ plus paclitaxel at triweekly intervals for a total of 2-4 cycles, with planned cumulative cisplatin dose of 180 to $200 \mathrm{mg} / \mathrm{m}^{2}$ in the. concurrent chemoradiation procedure. Part of patients received 1 or 2 cycles of adjuvant chemotherapy.

During the treatment, the patients underwent routine complete blood cell counts, blood-chemistry testing, and clinical assessment weekly. We recorded the important indicators, including hemoglobin (HLB), platelets (PLT), neutrophilic granulocyte percentage (NEU), at three stages: pre-treatment, in early stages of the treatment (after the beginning of the treatment about two weeks), and upon treatment completion respectively, as the potential prognostic factors. The evaluation and value of the main indicators were as follows: low HLB level, $\leq 90 \mathrm{~g} / \mathrm{L}$; low PLT level, $8100 \times 10^{9} / \mathrm{L}$ or high PLT level, $8350 \times 10^{9} / \mathrm{L}$; high NEU level, $\geq 0.8$, respectively.

Regarding treatment safety, the treatment was withheld in case of grade 4 hematologic toxicities or grade 3 to 4 nonhematologic toxicity, or fever $>38^{\circ} \mathrm{C}$, or severe liver and renal function damage. When the indicators mentioned above recovered to a normal level, the treatment was resumed. 


\section{Toxicities}

We also collected clinical data regarding treatment-related complications and toxicities. Complications occurring within 90 days from the beginning of the primary treatment were defined as acute complications, and those occurring later than 90 days were defined as late. The severity of acute complications was classified according to the $\mathrm{NCl}$ Common Terminology Criteria for Adverse Events, Version 3.0, and the late was graded according to the Radiation Therapy Oncology Group (RTOG) Late Radiation Morbidity Scoring Scheme.

\section{Follow-up}

Once the treatment was completed, patients were followed up every 3 or 4 months for the first 2 years, every 6 months for years 3 to 5 , and annually thereafter. During the follow-up period, besides general examinations and biology, US, CT, MRI of the abdomen and pelvis as well as PET/CT were optionally used for assessment. Overall survival (OS) was defined from the date of the treatment to death from any cause or the date of the last contact. Progression-free survival (PFS), on the other hand, was assessed from the completion of treatment to the date of disease progression or last contact.

\section{Statistical Analysis}

Survival rates were estimated and compared by the Kaplan-Meier method. A univariate Cox regression model was used to identify the predictors. The important variables were screened out with nonzero coefficients as potential predictors of this prediction model using the Elastic net method. Then multivariable Cox regression analysis was used to construct the predictive model based on the results of Elastic net regression, and a further nomogram was developed. Risk ratios and $95 \%$ confidence intervals (Cls) were calculated. The prediction efficiency of the predictive model was assessed by $C$-index with bootstrap verification (1000 bootstrap resampling), as well as calibration curves.

In addition, a risk stratification based on the level of nomogram scores was conducted, in which the population was divided into three subgroups (averaged as much as possible). To demonstrate whether the nomogram distinguishes between people at different risks, Kaplan-Meier curves were performed and validated for these three groups. All statistical analyses were conducted using R version 4.0.3 and SPSS 26.0 , and $p<0.05$ was considered to be statistically significant.

\section{Results}

\section{Patient Characteristics}

The tumor-associated and patients characteristics are summarized in Table 1. In all initial clinic visits, the main complaint was vaginal bleeding (93.9\%). The mean age was 53.4 years (22-75). The major 
histological subtype was squamous cell carcinoma (88.8\%), followed by adenocarcinoma (9.7\%). The distribution of $2018 \mathrm{FIGO}$ stage among the patients was as follows: stage $₫ \mathrm{~A}, 15.9 \%$; stage $\triangle \mathrm{B}, 41.5 \%$; stage $₫ A, 3.1 \%$; stage $\varangle B, 19.4 \%$; stage $\llbracket C, 15.1 \%$; stage $\varangle A, 5.0 \% .75 .2 \%$ of the patients had primary lesions with parametrial involvement. A total of $31.4 \%$ of the patients had pelvic lymph node enlargement on imaging. In addition, about half (50.8\%) presented bulky tumors (axis diameter larger or equal to $4 \mathrm{~cm}$ on imaging). During the treatment, five patients didn't complete the radiotherapy procedure owing to personal reasons. Most patients received chemotherapy with a cumulative cisplatin dose of 180 to 200 $\mathrm{mg} / \mathrm{m}^{2}$. and 28 patients received cisplatin-based chemotherapy with higher doses, including adjuvant chemotherapy. Out of 78 patients (30.2\%) were irradiated with 3DCRT using the four-field box irradiation, and out of $175(67.8 \%)$ with IMRT, totaling 46 cases receiving SIB for LNM. A total of 214 patients received ICBT. Because of chemo anaphylaxis, liver and kidney function deficiency, or personal rejection, 14 patients didn't undergo chemotherapy.

\section{Univariate and Multivariate Analysis of all Factors based on Elastic Net Regression}

Follow-up lasted until 31 October 2021 within a median follow-up of 47 months (3-71). At the analysis time, among 258 patients, 197 (76.4\%) were alive, out of which 177 (68.6\%) were alive free of disease. The 3-year and 5-year overall survivals were $73.7 \%$ and $65.9 \%$, respectively. While the 3 -year and 5-year progression-free survivals were $68.1 \%$ and $60.2 \%$. The 5 -year progression-free survival and overall survival of main factors were shown in Table 2.A total of 22 factors were analyzed as predictive prognostic factors, and the univariate analysis was presented in Table 1.

Elastic net regression analysis was performed to re-evaluate the variables in order to avoid the influence of confounding factors. Finally, 9 variables were retained with nonzero coefficients for PFS (Fig. 1 and Table 3) and 6 variables for OS (Fig. 2 and Table 3 ) as potential predictors of the prediction model. To get a more comprehensive view of the association between the survival outcome and these predictors, we further performed a multivariable Cox regression analysis and constructed a predictive model.

\section{Nomograms Establishment and Risk Stratification}

The results of the Cox regression analysis were given in Table $\mathbf{3}$ and visualized in the form of a nomogram plot to help practice in the clinic (Fig. 3). The C-index of the transfer nomogram were 0.671 for PFS, and 0.673 for OS, through 1000 repeated bootstrapping tests, respectively. As a consequence, different cumulative cisplatin dose ( $<180 \mathrm{mg} / \mathrm{m}^{2}$ : HR: $0.099,95 \% \mathrm{Cl}: 0.010-0.909, p=0.040 ; 180-$ 200mg/m²: HR: 0.058, 95\% Cl: 0.006-0.545, $p=0.012 ;>200 \mathrm{mg} / \mathrm{m}^{2}:$ HR: $0.050,95 \%$ Cl: 0.006-0.520, $p=0.012$ ), bulky tumors (HR: 1.869, 95\% Cl: 1.127-3.098, $p=0.015)$, ICBT (HR: 0.385, 95\% Cl: 0.218-0.681, $p=0.001)$, and thrombocytopenia pre-treatment (HR: 6.832, 95\% Cl: $1.169-39.902, p=0.032)$ had significant effect on progression-free survival. Overall survival was influenced by bulky tumors (HR: 1.916, 
95\% Cl: 1.100-3.336, $p=0.021)$, ICBT (HR: 0.499, 95\% Cl: 0.252-0.986, $p=0.045)$, thrombocytopenia pretreatment (HR: 3.865, 95\% Cl: 1.367-10.922, $p=0.010)$ and high neutrophilic granulocyte percentage level in early stages of the treatment (HR: $2.041,95 \% \mathrm{Cl}: 1.172-3.552, p=0.011)$. The calibration curves of the nomograms suggested relatively reliable agreements in both PFS and OS models (cross-validated concordance probability in Fig. 4).

After the nomogram score of each patient was calculated, all patients were divided into three groups according to their total score (PFS: low-risk group <42 points; intermediate-risk group 42-80 points; highrisk group $>80$ points, OS: low-risk group <35 points; intermediate-risk group 35-75 points; high-risk group $>75$ points). The 5-year PFS in the low-risk, intermediate-risk, and high-risk groups were $80.0 \%, 60.1 \%$, and $31.8 \%$, and the 5 -year OS were $83.1 \%, 51.8 \%$, and $38.5 \%$, all with a $p$-value $<0.05$, and Kaplan Meier curves were shown in Fig. 5.

\section{Toxicities}

Through clinical observation and follow-up, all of the acute and late toxicities are listed in Table 4. The common acute toxicities were rectitis(58.9\%); myelosuppression(83.7\%), as well as nausea or vomiting(87.6\%). Out of 51 patients (19.8\%) presented myelosuppression with grade 3 or higher. The most common late toxicity was rectitis occurring in a total of 84 patients (32.6\%). Most acute toxicities were acceptable with supportive clinical care. However, out of 7 patients were hospitalized again, requiring surgical intervention because of intestinal and rectum ulcers or continuous rectitis. Out of 2 cases were diagnosed as colonic perforation, and a single case died of an abdominal infection caused by perforation. One point to note is that all these cases showing severe toxicities received EBRT using 3DCRT during treatment. No grade 3 or worse toxicity of rectum and sigmoid was observed in patients with IMRT.

\section{Discussion}

In this study, we established and evaluated prognostic factors for locally advanced cervical cancer patients in southern China and their impact on both PFS and OS. In univariate analysis, 10 predictive factors showed statistical significance (bulky tumors; chemotherapy; chemotherapeutic agents; different cumulative cisplatin dose; ICBT; HLB in early stages of the treatment; PLT pre-treatment and in early stages of the treatment; NEU pre-treatment and in early stages of the treatment). Within the complete multivariable Cox regression, three predictors were confirmed significant for PFS (bulky tumors, ICBT, different cumulative cisplatin dose, thrombopenia pre-treatment,), and four significant predictors for OS (bulky tumors, different cumulative cisplatin dose, ICBT, high NEU level in early stages of the treatment). As a consequence, the nomograms for 3-year and 5-year survival were constructed with all the significant factors.

Moreover, we further took the nomogram score into the evaluation to perform different risk stratification. The subsequent Kaplan-Meier analysis showed a significant difference in survival time between three 
groups of stratification, suggesting that the nomogram can distinguish between patients at different risks. The 5-year PFS in the low-risk, intermediate-risk, and high-risk group were $80.0 \%, 60.1 \%$, and $31.8 \%$, and the 5 -year OS were $83.1 \%, 51.8 \%$, and $38.5 \%$, respectively. Considering the obviously lower survival rate in the high-risk group, more individualized and intense treatment may be required for this group of patients.

As is reported, age is closely related to the prognosis of patients with LACC, especially patients younger than 35 years old, which were considered to be more susceptible to local recurrence or distant metastasis ${ }^{9}$. Nevertheless, our study didn't reveal that age has a statistically significant effect on OS or PFS, probably due to the lack of detailed stratification and fewer patients in the younger age group. As for pathological type, with an increasing incidence in recent years, adenocarcinoma is considered a poor prognostic factor in patients with $\mathrm{LACC}^{10}$. Compared with SCC, adenocarcinoma is more likely to make larger primary lesions and lead to distant metastasis. However, Galic Vijaya et al. reported that despite the lower complete remission rate of $86.5 \%$, patients with adenocarcinoma receiving CCRT had no apparent difference in long-term survival ${ }^{11}$. Likewise, our study found that patients who received CCRT have no statistical difference in OS and PFS between adenocarcinoma and SCC.

As is known, pelvic and para-aortic LNM plays a key role in the poor prognosis of cervical cancer. Liu et al. indicated that the overall survival was $91 \%$ for pelvic LNM negative cohort and $67 \%$ for that positive cohort $^{12}$, Most nomograms estimating PFS or OS for LACC include pelvic lymph node status as a significant risk factor, regardless of diagnostic methods: lymphadenectomy, PET/CT, or multiple imaging $^{8,13}$. In 2018, FIGO revised the 2014 system into the 2018 staging system of cervical cancer ${ }^{14}$. One crucial difference from the previous staging system is that the latest 2018 system designates patients with regional lymph node metastasis into new stage $₫$ category, in which patients with pelvic lymph node metastasis only are allocated to stage $₫ \mathrm{C} 1$, and with positive para-aortic lymph node (PALN) allocated to stage $\mathbb{X C 2}$. Our study comprehensively evaluated patients diagnosed with the previous staging system before 2018 and restaged those who met the $\varangle \mathrm{C}$ category in $2018 \mathrm{FIGO}$. A total of 38 were diagnosed as $\mathbb{} \mathrm{C}$. However, we failed to analyze that the FIGO stage, the $\mathbb{C}$ category in a particular, is a significant and independent factor, and there is no significant difference in 5-year overall survival between $\varangle \mathrm{C}$ and $\varangle \mathrm{B}(59.9 \%$ vs. $54.8 \%, p>0.05)$. What calls for attention is that stage $\mathbb{X C}$ of the new system doesn't take primary tumor size and extent as well as the characteristics of LNM into consideration. Liu et al. ${ }^{15}$ reported that tumor size and number of PLNM are significant prognostic factors for DFS in patients with stage $\mathbb{X} 1 \mathrm{r}$ while confirming the heterogeneity among this group of patients. Therefore, regarding patients with combined and complicated status of large tumor size, lymph node metastasis, or other potential risk factors, the reasonability of new FIGO staging system to evaluate prognosis is limited.

On the other hand, the comparability of clinical stages in different regions and countries in the world has decreased, because the clinical stage in some countries, developed countries especially, is affected by imaging like MRI or PET/CT. In a meta-analysis, PET/CT and DWI-MRI have a high accuracy in detecting LNM. Among several modalities, PET/CT has the highest specificity, and DWI-MRI has the highest 
sensitivity ${ }^{16,17}$. What's more, compared to MRI on regional hypogastrium or pelvic, PET/CT has its advantages on detecting long-distant LNMs. Henrik Hansen et al. ${ }^{16}$ reported that the inclusion of PET/CT in the pre-radiotherapy diagnostic protocol correlated with survival benefits after CCRT in node-negative cervical cancer patients of $23 \%, 19 \%$ for OS, and disease-free survival (DFS). Due to the imbalance of regional development, the diagnoses of LACC patients in less developed areas were relatively limited. In our study, only 18 patients were diagnosed as stage $\mathbb{}$ C through PET/CT. A majority of patients refused to perform PET/CT or regional DWI-MRI because of high cost and personal reasons, which possibly lead to underestimates of clinical stage in patients with potential pelvic and para-aortic lymph node metastasis or worse distant organ metastasis. With developments in techniques, the failure pattern of LACC has changed. Lymph node failure has become a sort of main failure site remaining unsolved. It would be necessary to use high-precision imaging equipment like PET/CT or DWI-MRI to define the clinical metastases exactly in future practice.

Large tumor size was strongly suggested as a prognostic factor for $\mathrm{LACC}^{18}$. Similarly, our study demonstrated that patients with bulky tumors had an adverse effect on PFS as well as OS. It is acknowledged that tumor volume is the most direct indicator of tumor burden, representing the number of clones needed to be killed among the tumor ${ }^{19}$. In addition, large tumor size tends to give rise to a lack of oxygen, radiation resistance, and worse local control. In the relatively less-developed region of southern China, plenty of patients were diagnosed as advanced stages with the large primary site before treatment, leading to poor prognosis.

With advances in Image-guided radiation therapy (IGRT) and techniques, IMRT has gradually taken the place of traditional two-dimensional (2D) and three-dimensional (3D) techniques in EBRT procedure for better radiation protection to surrounding organs, bone marrow sparing as well as lower toxicity 20,21 . As shown in our study, patients with IMRT had a lower incidence of toxicity compared to those with four-box field, especially severe and unacceptable rectum and sigmoid toxicity. Nevertheless, we failed to observe that IMRT improved overall survival significantly, which may be attributable to the slightly short time follow-up (median of 47 months), representing the low number of patients followed beyond 5 years.

For decades, it is universally acknowledged that the combination of radiotherapy with chemotherapy prolongs disease-free survival while reducing mortality. In our study, chemotherapy was performed using cisplatin alone or with several combinations, and most patients received standard chemotherapy performed at weekly or tri-weekly intervals with the cumulative cisplatin dose of 180 to $200 \mathrm{mg} / \mathrm{m}^{2}$. Interestingly, we observed that not chemotherapy regimens but different cumulative cisplatin doses had an impact on PFS $(p<0.05)$. Except for those who didn't complete chemotherapy as planned, patients who received higher doses of cisplatin had a better 5 -year PFS (74.4\% vs $67.0 \%)$. Due to the higher incidence of bulky tumor (50.8\%) and parametrial involvement (75.2\%) among the enrolled cohort, it seemed that dose-intensive or additional adjuvant cisplatin-based chemotherapy not only inhibits accelerated tumor cell repopulation in a period but also further reduces potential systemic micro-metastases, thereby probably increasing the actual local control rate. Likewise, Landoni et al. ${ }^{22}$ suggested that additional 
chemotherapy could benefit those patients with intra-cervical residual and suboptimal responses. However, our study did not demonstrate that chemotherapy with higher doses of cisplatin benefited the overall survival of LACC patients, which probably was associated with the higher incidence of hematological toxicity in additional chemotherapy. Therefore, among LACC patients at different risks, it is necessary initially to re-evaluated the best-fit administration and delivery of chemotherapy.

Recently, indicators of blood cell counts have attracted attention as potential prognostic factors in cancer patients. In contrast to the nomograms performed by Rose et $\mathrm{al}^{8}$, we obtained three main complete indicators of blood cell counts at different periods during the treatment, aiming to investigate their dynamic variations and analyzed their prognostic predictive value, in consequence finding that thrombopenia before the treatment had adverse effects on both PFS $(p=0.032)$ and OS $(p=0.010)$, and high NEU level in early stages of the treatment was associated to poor OS $(p=0.011)$. As is identified as a marker of poor prognosis in several forms of cancer, thrombocytopenia represents coagulation disorders and a higher incidence of bleeding risk, potentially leading to less tolerance of CCRT ${ }^{23}$. The tumor microenvironment plays a key role in growth and metastasis in present studies, which can be visualized by varieties of inflammation-related cells, such as neutrophils, platelets, and lymphocytes, especially neutrophils ${ }^{24}$. Continuous high level of NEU is one of the paracancerous syndromes of many malignant tumors, representing chronic inflammation in patients. Inflammation could change the systemic microenvironment for inducing the angiogenesis of tumors. What's more, Inflammation level positively correlated with granulocyte colony-stimulating factor (G-CSF) expression in tumor tissues, which stimulates the production of myeloid-derived suppressor cell (MDSC), thus leading to the rapid development of the tumor and radiation resistance ${ }^{24}$. Yet there is little high-quality evidence on how to eliminate such chronic inflammatory responses in oncology patients.

In the current study, multivariate analyses were performed based on the elastic net regression model as the best-fit model to select potential risk factors. Moreover, nomograms and risk stratification were established to verify our predictive model accuracy further. As a result, the calibration plots showed an acceptable consistency between the predictive and actual probability of the survivals (cross-validated concordance probability $\mathrm{c}=0.684$ for PFS, and 0.654 for OS), which could be the study's strengths. However, as this is a retrospective observational study, some limitations need to be addressed. First, the follow-up time was slightly short (median of 47 months). Second, not all patients benefited from a uniform and standard therapeutic scheme. Third, all patients were enrolled from a single institution. The factors mentioned above represent the fact that several biases would be inevitable to occur. Therefore, these factors can only be eliminated in prospective randomized studies with a more stratified and larger population.

\section{Conclusion}

Cisplatin-based concurrent chemoradiation revealed a favorable survival of LACC in southern China. In addition to frequent factors (bulky tumors, ICBT), cumulative cisplatin dose and neutrophil percentage seem to be essential outcome predictors. The present study shows the prognostic value of nomogram to 
predict tumor-related survival and establish risk stratification in a setting of LACC patients from southern China. Therefore, future individualized tailoring of therapy should focus even further on the high-risk patient populations.

\section{Declarations}

\section{Funding}

This study was supported by the Guangxi Zhuang Autonomous Region Health and Wellness Committee Science and Technology Project (S2017017). The funders had no role in study design, data collection and analysis, decision to publish, or preparation of the manuscript.

\section{Competing interests}

Conflict of interest relevant to this article was not reported.

\section{Availability of data and material}

The datasets generated during the current study are not publicly available due to ethical considerations, but are available from the corresponding author on reasonable request.

\section{Authors' contributions}

$\mathrm{XL}, \mathrm{LH}$, and LJ participated in the conceptualization, data curation, and writing the original draft. SM and MS contributed to validation of the results. YQ, JQ, and QN developed the methodology and performed formal analysis. YZ reviewed and edited the manuscript and performed project administration. FW supervised the research. All authors contributed to the article and approved the submitted version.

\section{Ethics approval}

The authors state that they have obtained appropriate institutional review board approval or have followed the principles outlined in the Declaration of Helsinki for all human or animal experimental investigations.

\section{Consent to participate}

Written informed consent was obtained from the patients. 


\section{Consent for publication}

Not applicable.

\section{Acknowledgments}

The authors would like to gratefully acknowledge the patients who participated in the study.

\section{References}

1. Sung, H.et al. Global Cancer Statistics 2020: GLOBOCAN Estimates of Incidence and Mortality Worldwide for 36 Cancers in 185 Countries. CA Cancer J Clin 71, 209-249, doi:10.3322/caac.21660 (2021).

2. Gu, X. Y.et al. [Incidence and mortality of cervical cancer in China, 2014]. Zhonghua Zhong Liu Za Zhi 40, 241-246, doi:10.3760/cma.j.issn.0253-3766.2018.04.001 (2018).

3. Whitney, C. W.et al. Randomized comparison of fluorouracil plus cisplatin versus hydroxyurea as an adjunct to radiation therapy in stage IIB-IVA carcinoma of the cervix with negative para-aortic lymph nodes: a Gynecologic Oncology Group and Southwest Oncology Group study. J Clin Oncol 17, 13391348, doi:10.1200/JC0.1999.17.5.1339 (1999).

4. Rose, P. G.et al. Concurrent cisplatin-based radiotherapy and chemotherapy for locally advanced cervical cancer. N Engl J Med 340, 1144-1153, doi:10.1056/NEJM199904153401502 (1999).

5. Rose, P. G.et al. Long-term follow-up of a randomized trial comparing concurrent single agent cisplatin, cisplatin-based combination chemotherapy, or hydroxyurea during pelvic irradiation for locally advanced cervical cancer: a Gynecologic Oncology Group Study. J Clin Oncol 25, 2804-2810, doi:10.1200/JC0.2006.09.4532 (2007).

6. Morris, M.et al. Pelvic radiation with concurrent chemotherapy compared with pelvic and para-aortic radiation for high-risk cervical cancer. N Engl J Med 340, 1137-1143, doi:10.1056/NEJM199904153401501 (1999).

7. Chemoradiotherapy for Cervical Cancer Meta-Analysis, C. Reducing uncertainties about the effects of chemoradiotherapy for cervical cancer: a systematic review and meta-analysis of individual patient data from 18 randomized trials. J Clin Oncol 26, 5802-5812, doi:10.1200/JC0.2008.16.4368 (2008).

8. Rose, P. G.et al. Nomograms Predicting Progression-Free Survival, Overall Survival, and Pelvic Recurrence in Locally Advanced Cervical Cancer Developed From an Analysis of Identifiable Prognostic Factors in Patients From NRG Oncology/Gynecologic Oncology Group Randomized Trials of Chemoradiotherapy. J Clin Oncol 33, 2136-2142, doi:10.1200/JC0.2014.57.7122 (2015).

9. Wang, J.et al. Patient age, tumor appearance and tumor size are risk factors for early recurrence of cervical cancer. Mol Clin Oncol 3, 363-366, doi:10.3892/mco.2014.465 (2015). 
10. Rose, P. G.et al. Locally advanced adenocarcinoma and adenosquamous carcinomas of the cervix compared to squamous cell carcinomas of the cervix in gynecologic oncology group trials of cisplatin-based chemoradiation. Gynecol Oncol 135, 208-212, doi:10.1016/j.ygyno.2014.08.018 (2014).

11. Galic, V.et al. Prognostic significance of adenocarcinoma histology in women with cervical cancer. Gynecol Oncol 125, 287-291, doi:10.1016/j.ygyno.2012.01.012 (2012).

12. Liu, Y.et al. The Number of Positive Pelvic Lymph Nodes and Multiple Groups of Pelvic Lymph Node Metastasis Influence Prognosis in Stage IA-IIB Cervical Squamous Cell Carcinoma. Chin Med J (Engl) 128, 2084-2089, doi:10.4103/0366-6999.161372 (2015).

13. Wang, W., Liu, X., Meng, Q., Zhang, F. \& Hu, K. Nomograms predicting survival and patterns of failure in patients with cervical cancer treated with concurrent chemoradiotherapy: A special focus on lymph nodes metastases. PLoS One 14, e0214498, doi:10.1371/journal.pone.0214498 (2019).

14. Bhatla, N., Aoki, D., Sharma, D. N. \& Sankaranarayanan, R. Cancer of the cervix uteri. Int J Gynaecol Obstet 143 Suppl 2, 22-36, doi:10.1002/ijgo.12611 (2018).

15. Liu, X.et al. A Risk Stratification for Patients with Cervical Cancer in Stage IIIC1 of the 2018 FIGO Staging System. Sci Rep 10, 362, doi:10.1038/s41598-019-57202-3 (2020).

16. Hansen, H. V.et al. Survival outcomes in patients with cervical cancer after inclusion of PET/CT in staging procedures. Eur J Nucl Med Mol Imaging 42, 1833-1839, doi:10.1007/s00259-015-3113-7 (2015).

17. Liu, B., Gao, S. \& Li, S. A Comprehensive Comparison of CT, MRI, Positron Emission Tomography or Positron Emission Tomography/CT, and Diffusion Weighted Imaging-MRI for Detecting the Lymph Nodes Metastases in Patients with Cervical Cancer: A Meta-Analysis Based on 67 Studies. Gynecol Obstet Invest 82, 209-222, doi:10.1159/000456006 (2017).

18. Wakayama, A.et al. Concurrent chemoradiotherapy for non-bulky stage IB/II cervical cancer without pelvic node enlargement. Anticancer Res 33, 5123-5126 (2013).

19. He, Y. X.et al. Prognostic value and predictive threshold of tumor volume for patients with locally advanced nasopharyngeal carcinoma receiving intensity-modulated radiotherapy. Chin J Cancer 35, 96, doi:10.1186/s40880-016-0159-2 (2016).

20. Erpolat, O. P.et al. Comparison of hematologic toxicity between 3DCRT and IMRT planning in cervical cancer patients after concurrent chemoradiotherapy: a national multi-center study. Eur J Gynaecol Oncol 35, 62-66 (2014).

21. Chuong, M. D.et al. Intensity-Modulated Radiation Therapy vs. 3D Conformal Radiation Therapy for Squamous Cell Carcinoma of the Anal Canal. Gastrointest Cancer Res 6, 39-45 (2013).

22. Landoni, F.et al. Is there a role for postoperative treatment in patients with stage Ib2-llb cervical cancer treated with neo-adjuvant chemotherapy and radical surgery? An Italian multicenter retrospective study. Gynecol Oncol 132, 611-617, doi:10.1016/j.ygyno.2013.12.010 (2014).

23. Wang, H.et al. Combined PLT and NE to predict the prognosis of patients with locally advanced cervical cancer. Sci Rep 10, 11210, doi:10.1038/s41598-020-66387-x (2020). 
24. Quail, D. F. \& Joyce, J. A. Microenvironmental regulation of tumor progression and metastasis. Nat Med 19, 1423-1437, doi:10.1038/nm.3394 (2013).

\section{Tables}

\section{Table 1}

Characteristics for patients and results of univariate regression analysis. 
Factors

No.(\%) PFS

OS

$\mathrm{HR}$

$p$-value HR

$p$-value

$\mathrm{n}=258 \quad 95 \% \mathrm{Cl}$

$\begin{array}{llll}\text { Age } & 20(7.8 \%) & \mathrm{R} & \mathrm{R}\end{array}$

$\leq 40$

$41-59$

$170(65.9 \%)$

0.634

$(0.301-$

0.231

0.602

(0.253- $\quad 0.252$

(3)
$1.433)$

$\geq 60$

68(26.4\%)

0.738

$(0.330$
$1.650)$

0.459

0.662

$(0.257-\quad 0.393$

1.707)

Pathological_type

SCC

229(88.8\%)

$\mathrm{R}$

R

Adenocarcinoma

$25(9.7 \%)$

$\begin{array}{ll}0.749 \quad(0.326- \\ & 1.722)\end{array}$

0.496

0.353

(0.0861.447)

0.148

Others

$4(1.6 \%)$

$1.423 \quad(0.349-$

$0.622 \quad 1.048$

(0.145- 0.963

7.579)

FIGO_stage

\begin{tabular}{|c|c|c|c|c|c|c|c|}
\hline$\nabla \mathrm{A}$ & $41(15.9 \%)$ & $\mathrm{R}$ & & & $\mathrm{R}$ & & \\
\hline$\otimes \mathrm{B}$ & $107(41.5 \%)$ & 0.849 & $\begin{array}{l}(0.052- \\
2.884)\end{array}$ & 0.356 & 0.883 & $\begin{array}{l}(0.028- \\
1.619)\end{array}$ & 0.135 \\
\hline$\triangle A$ & $8(3.1 \%)$ & 0.781 & $\begin{array}{l}(0.032- \\
3.970)\end{array}$ & 0.402 & 0.542 & $\begin{array}{l}(0.008- \\
2.118)\end{array}$ & 0.152 \\
\hline $\mathbb{\nabla B}$ & $50(19.4 \%)$ & 1.600 & $\begin{array}{l}(0.098- \\
5.491)\end{array}$ & 0.763 & 1.438 & $\begin{array}{l}(0.044- \\
2.687)\end{array}$ & 0.310 \\
\hline$\triangle C^{*}$ & $39(15.1 \%)$ & 1.895 & $\begin{array}{l}(0.115- \\
6.565)\end{array}$ & 0.891 & 1.425 & $\begin{array}{l}(0.042- \\
2.765)\end{array}$ & 0.314 \\
\hline$\nabla \mathrm{A}$ & $13(5.0 \%)$ & 1.199 & $\begin{array}{l}(0.064- \\
4.734)\end{array}$ & 0.585 & 1.658 & $\begin{array}{l}(0.045- \\
3.488)\end{array}$ & 0.405 \\
\hline
\end{tabular}

Bulky tumors

No

$127(49.2 \%)$

R

R

Yes

131(50.8\%) $\quad 1.989$

(1.268-

3.119)

$\mathbf{0 . 0 0 3} 1.965$

(1.1443.377)

Pelvic lymph node enlargement

No

177(68.6\%)

$\mathrm{R}$

$\mathrm{R}$ 
$\begin{array}{llllllll}\text { Yes } & 81(31.4 \%) & 1.290 & \begin{array}{c}(0.817- \\ 2.038)\end{array} & 0.275 & 1.039 & (0.581- & 0.898 \\ & & & & & 1.857) & \end{array}$

Parametrial

involvement

\begin{tabular}{llllllll}
\hline No & $64(24.8 \%)$ & $\mathrm{R}$ & \multicolumn{5}{c}{$\mathrm{R}$} \\
\hline Yes & $194(75.2 \%)$ & 1.218 & $\begin{array}{l}(0.735- \\
2.018)\end{array}$ & 0.445 & 1.034 & $(0.578-$ & 0.911 \\
& & & & & $1.849)$ &
\end{tabular}

Radiotherapy

completion

\begin{tabular}{llllllll} 
No & $5(1.9 \%)$ & $\mathrm{R}$ & \multicolumn{5}{c}{$\mathrm{R}$} \\
Yes & $253(98.1 \%)$ & 0.861 & $\begin{array}{l}(0.211- \\
3.503)\end{array}$ & 0.834 & 0.573 & $(0.140-$ & 0.440 \\
& & & & & $2.355)$ &
\end{tabular}

Way of EBRT

\begin{tabular}{llllllll} 
No completion & $5(1.9 \%)$ & $\mathrm{R}$ & \multicolumn{5}{c}{$\mathrm{R}$} \\
IMRT & $175(67.8 \%)$ & 0.752 & $\begin{array}{l}(0.183- \\
3.097)\end{array}$ & 0.693 & 0.530 & $\begin{array}{l}(0.127- \\
2.208)\end{array}$ & 0.383 \\
3DCRT & $78(30.2 \%)$ & 1.100 & $\begin{array}{l}(0.263- \\
4.594)\end{array}$ & 0.896 & 0.667 & $\begin{array}{l}(0.156- \\
2.857)\end{array}$ & 0.585 \\
\hline
\end{tabular}

SIB for LNM

\begin{tabular}{llllllll}
\hline No & $215(82.2 \%)$ & $\mathrm{R}$ & \multicolumn{5}{c}{$\mathrm{R}$} \\
Yes & $46(17.8 \%)$ & 0.871 & $\begin{array}{l}(0.471- \\
1.610)\end{array}$ & 0.659 & 0.778 & $(0.351-$ & 0.536 \\
& & & & & $1.724)$ & \\
\hline
\end{tabular}

Chemotherapy

\begin{tabular}{llllllll} 
No & $14(5.4 \%)$ & $R$ & \multicolumn{7}{c}{$\mathrm{R}$} \\
Yes & $244(95.6 \%)$ & 0.356 & $\begin{array}{c}(0.199- \\
0.638)\end{array}$ & 0.001 & 0.326 & $(0.163-$ & 0.002
\end{tabular}

Chemotherapeutic agents

\begin{tabular}{|c|c|c|c|c|c|c|c|}
\hline No chemo & $14(5.4 \%)$ & $\mathrm{R}$ & & & $\mathrm{R}$ & & \\
\hline Weekly cisplatin & 103(39.9\%) & 0.253 & $\begin{array}{l}(0.119- \\
0.536)\end{array}$ & $<0.001$ & 0.167 & $\begin{array}{l}(0.071- \\
0.393)\end{array}$ & $<0.001$ \\
\hline Cisplatin and 5-FU & $71(27.5 \%)$ & 0.504 & $\begin{array}{l}(0.245- \\
1.036)\end{array}$ & 0.062 & 0.347 & $\begin{array}{l}(0.156- \\
0.771)\end{array}$ & 0.009 \\
\hline $\begin{array}{l}\text { Cisplatin plus } \\
\text { paclitaxel }\end{array}$ & $52(20.2 \%)$ & 0.411 & $\begin{array}{l}(0.129- \\
1.314)\end{array}$ & 0.134 & 0.454 & $\begin{array}{l}(0.122- \\
1.692)\end{array}$ & 0.239 \\
\hline Others & $18(7.0 \%)$ & 0.375 & $(0.170-$ & 0.015 & 0.298 & $(0.125-$ & 0.006 \\
\hline
\end{tabular}


C. C. D $\left(\mathrm{mg} / \mathrm{m}^{2}\right)$

\begin{tabular}{|c|c|c|c|c|c|c|c|}
\hline 0 & $14(5.4 \%)$ & $\mathrm{R}$ & & & $\mathrm{R}$ & & \\
\hline$<180$ & $44(17.0 \%)$ & 0.423 & $\begin{array}{l}(0.201- \\
0.889)\end{array}$ & 0.023 & 0.515 & $\begin{array}{l}(0.225- \\
1180)\end{array}$ & 0.117 \\
\hline $180-200$ & $172(66.7 \%)$ & 0.261 & $\begin{array}{l}(0.137- \\
0.496)\end{array}$ & $<0.001$ & 0.244 & $\begin{array}{l}(0.115- \\
0.518)\end{array}$ & $<0.001$ \\
\hline$>200$ & $28(10.9 \%)$ & 0.284 & $\begin{array}{l}(0.112- \\
0.724)\end{array}$ & 0.008 & 0.354 & $\begin{array}{l}(0.125- \\
0.999)\end{array}$ & 0.049 \\
\hline
\end{tabular}

ICBT

No

$44(17.1 \%)$

$\mathrm{R}$

$\mathrm{R}$

Yes

$214(82.9 \%)$

0.407

$(0.242-$

$0.687)$

$\mathbf{0 . 0 0 1} \quad 0.481$

$(0.242-$

$0.954)$

0.036

HLB pre-treatment

Normal

213(82.6\%)

$\mathrm{R}$

$45(17.4 \%)$

1.375

$(0.815-$

2.320

(0.868-

2.913)

0.133

HLB in early stages of the treatment

\begin{tabular}{|c|c|c|c|c|c|c|c|}
\hline Normal & 193(74.8\%) & $\mathrm{R}$ & & & $\mathrm{R}$ & & \\
\hline Low & $65(25.2 \%)$ & 1.338 & $\begin{array}{l}(0.836- \\
2.142)\end{array}$ & 0.225 & 1.794 & $\begin{array}{l}(1.044- \\
3.083)\end{array}$ & 0.034 \\
\hline
\end{tabular}

HLB upon treatment

completion

Normal

$187(72.5 \%)$

R

$71(27.5 \%)$

1.052

(0.653-

1.695)

$\mathrm{R}$

Low

71

\section{PLT pre-treatment}

Normal

High

Low

$206(79.8 \%)$

$\mathrm{R}$

$\begin{array}{llll} & 1.413 \quad \begin{array}{l}(0.824- \\ 2.421)\end{array} & 0.209 & 1.506\end{array}$

4.437

$(1.778-$
$11.070)$

0.001 $\begin{array}{lll}1.473 & \begin{array}{l}(0.857- \\ 2.532)\end{array} & 0.162\end{array}$

PLT in early stages of the treatment

Normal

$$
227(88.0 \%)
$$

R

R 
High

$21(8.1 \%)$

1.299

(0.624-

0.485

1.398

(0.595-

0.442

2.705)

3.285)

Low

$10(3.9 \%)$

3.199

(1.466-

0.003

3.376

(1.435-

7.942)

0.005

PLT upon treatment

completion

\begin{tabular}{|c|c|c|c|c|c|c|c|}
\hline Normal & 233(90.3\%) & $\mathrm{R}$ & & & $\mathrm{R}$ & & \\
\hline High & $6(2.3 \%)$ & 1.356 & $\begin{array}{l}(0.427- \\
4.308)\end{array}$ & 0.606 & 1.309 & $\begin{array}{l}(0.318- \\
5.389)\end{array}$ & 0.709 \\
\hline Low & $19(7.4 \%)$ & 1.806 & $\begin{array}{l}(0.901- \\
3.619)\end{array}$ & 0.096 & 1.664 & $\begin{array}{l}(0.711- \\
3.897)\end{array}$ & 0.240 \\
\hline
\end{tabular}

NEU pre-treatment

Normal

231(89.5\%)

$\mathrm{R}$

$\mathrm{R}$

High

$27(10.5 \%)$

1.523

(0.807-

0.195

2.453

(1.267-

4.750)

0.008 2.877)

R

2.122

$(1.363-$
$3.303)$

R

High

$75(29.1 \%)$

3.303)

$0.001 \quad 2.586$

$(1.520-\quad<0.001$ 4.402)

NEU upon treatment completion

Normal 232(89.9\%)

R

$\mathrm{R}$

High

26(10.1\%)

1.107

(0.644-

1.903)

0.714

1.736

(0.530 2.382)

R Reference; FIGO Federation International of Gynecology and Obstetrics; SCC squamous cell carcinoma; LNM lymph node metastasis; C.C.D cumulative cisplatin dose. EBRT external beam radiation therapy; IMRT intensity-modulated radiotherapy; ICBT intracavitary brachytherapy; HLB hemoglobin; PLT platelets; NEU neutrophilic granulocyte percentage.

*Out of 37 patients diagnosed as $₫ C 1 r$, out of 2 patients were diagnosed as $₫ C 2 r$ according to 2018 FIGO guidelines. Out of 18 patients were diagnosed through PET/CT, the others through CT or MRI.

\section{Table 2}

5-year PFS and OS of main factors 
Factors 5-year PFS \%SE 5-year OS \%SE

FIGO_stage

\begin{tabular}{lllll}
\hline$\square \mathrm{A}$ & 62.0 & 10.2 & 66.5 & 10.7 \\
\hline$\triangle \mathrm{B}$ & 69.2 & 5.5 & 74.7 & 5.3 \\
\hline$\triangle \mathrm{A}$ & 65.6 & 20.9 & 75.0 & 21.7 \\
\hline$\triangle \mathrm{B}$ & 52.1 & 8.8 & 54.8 & 9.8 \\
\hline$\square \mathrm{C}$ & 44.6 & 11.5 & 59.9 & 13.0 \\
\hline$\square \mathrm{A}$ & 33.7 & 24.8 & 35.5 & 27.4
\end{tabular}

Bulky tumors

\begin{tabular}{lllll} 
No & 71.5 & 4.8 & 76.0 & 4.8 \\
\hline Yes & 46.6 & 6.4 & 52.4 & 6.9
\end{tabular}

Way of EBRT

\begin{tabular}{lllll}
\hline IMRT & 65.8 & 4.6 & 70.3 & 4.6 \\
\hline 3DCRT & 51.0 & 6.8 & 59.9 & 7.5 \\
\hline
\end{tabular}

IGBT

$\begin{array}{lllll}\text { No } & 33.6 & 12.7 & 38.2 & 17.8 \\ \text { Yes } & 64.1 & 4.1 & 68.7 & 4.2\end{array}$

C. C. D $\left(\mathrm{mg} / \mathrm{m}^{2}\right)$

\begin{tabular}{lllll}
0 & 11.7 & 10.1 & 12.2 & 11.2 \\
\hline$<180$ & 44.7 & 11.2 & 47.4 & 11.7 \\
\hline $180-200$ & 67.0 & 4.5 & 74.5 & 4.6 \\
\hline$>200$ & 74.4 & 8.4 & 76.8 & 8.4 \\
\hline PLT pre-treatment & & & & \\
\hline Normal & 62.5 & 4.4 & 68.2 & 4.6 \\
\hline High & 56.3 & 8.7 & 65.5 & 9.0 \\
\hline Low & 16.7 & 15.2 & 16.7 & 15.2 \\
\hline
\end{tabular}

NEU in early stages of the treatment

$\begin{array}{lllll}\text { Normal } & 67.1 & 4.5 & 73.4 & 4.6\end{array}$


$\begin{array}{lllll}\text { High } & 43.4 & 7.5 & 48.5 & 7.9\end{array}$

SE, standard error.

\section{Table 3}

Results of multivariate Cox regression analysis for PFS and OS 
Variables for PFS HR $\quad 95 \% \mathrm{Cl} \quad p$-value

FIGO_stage

\begin{tabular}{llll}
\hline$\varangle \mathrm{B}$ & 0.939 & $0.452-1.951$ & 0.867 \\
\hline$\triangle \mathrm{A}$ & 1.053 & $0.229-4.838$ & 0.946 \\
\hline$\varangle \mathrm{B}$ & 1.382 & $0.618-3.092$ & 0.429 \\
\hline$\varangle \mathrm{C}$ & 1.800 & $0.785-4.126$ & 0.164 \\
\hline$\varangle \mathrm{A}$ & 1.020 & $0.331-3.138$ & 0.971 \\
\hline Bulky tumors & 1.869 & $1.127-3.098$ & $\mathbf{0 . 0 1 5}$ \\
\hline Way of EBRT & & & \\
\hline IMRT & & & \\
\hline Four-field box & 0.624 & $0.122-3.187$ & 0.571 \\
\hline Chemotherapy & 1.067 & $0.209-5.455$ & 0.937 \\
\hline C. C. $D\left(\mathrm{mg} / \mathrm{m}^{2}\right)$ & 4.048 & $0.501-32.679$ & 0.189 \\
\hline$<180$ & & & \\
\hline 180-200 & & & \\
\hline$>200$ & 0.099 & $0.010-0.909$ & $\mathbf{0 . 0 4 0}$ \\
\hline ICBT & 0.058 & $0.006-0.545$ & $\mathbf{0 . 0 1 2}$ \\
\hline
\end{tabular}

PLT pre-treatment

\begin{tabular}{llll}
\hline High & 1.787 & $0.940-3.397$ & 0.076 \\
\hline Low & 6.832 & $1.169-39.902$ & $\mathbf{0 . 0 3 2}$
\end{tabular}

PLT in early stages of the treatment

\begin{tabular}{llll}
\hline High & 0.716 & $0.309-1.658$ & 0.435 \\
\hline Low & 0.871 & $0.193-3.918$ & 0.857 \\
\hline High NEU level in early stages & 1.541 & $0.934-2.542$ & 0.090 \\
\hline Variables for OS & HR & $95 \% \mathrm{Cl}$ & $p$-value \\
\hline Bulky tumors & 1.916 & $1.100-3.336$ & $\mathbf{0 . 0 2 1}$ \\
\hline Chemotherapy & 1.596 & $0.203-12.523$ & 0.656
\end{tabular}


C. C. D $\left(\mathrm{mg} / \mathrm{m}^{2}\right)$

\begin{tabular}{llll}
\hline$<180$ & 0.338 & $0.038-2.980$ & 0.329 \\
\hline $180-200$ & 0.177 & $0.019-1.591$ & 0.122 \\
\hline$>200$ & 0.192 & $0.018-1.990$ & 0.166 \\
\hline ICBT & 0.499 & $0.252-0.986$ & $\mathbf{0 . 0 4 5}$
\end{tabular}

PLT pre-treatment

\begin{tabular}{llll}
\hline High & 1.408 & $0.698-2.837$ & 0.338 \\
\hline Low & 3.865 & $1.367-10.922$ & $\mathbf{0 . 0 1 0}$ \\
\hline High NEU level in early stages & 2.041 & $1.172-3.552$ & $\mathbf{0 . 0 1 1}$
\end{tabular}

Table 4

Acute and late toxicities for enrolled patients $(n=258)$

\begin{tabular}{|llllll|}
\hline Acute toxicities & \multicolumn{3}{l}{ Grade } \\
\hline Toxicity & G0 & G1 & G2 & G3 & G4 \\
\hline Rectum and sigmoid & 106 & 123 & 28 & 1 & 0 \\
\hline Myelosuppression & 42 & 50 & 101 & 48 & 3 \\
\hline Bladder & 181 & 75 & 2 & 0 & 0 \\
\hline Skin & 190 & 66 & 2 & 0 & 0 \\
\hline Nausea or vomiting & 32 & 160 & 66 & 0 & 0 \\
\hline Weight loss & 156 & 95 & 7 & 0 & 0 \\
\hline Late toxicities & Grade (RTOG/EORTC) & \\
\hline Toxicity & 0 & 1 & 2 & 3 & 4 \\
\hline Rectum and sigmoid & 174 & 62 & 15 & 5 & 2 \\
\hline Small intestine & 215 & 38 & 15 & 0 & 0 \\
\hline Bladder & 220 & 38 & 0 & 0 & 0 \\
\hline
\end{tabular}

Figures 
A

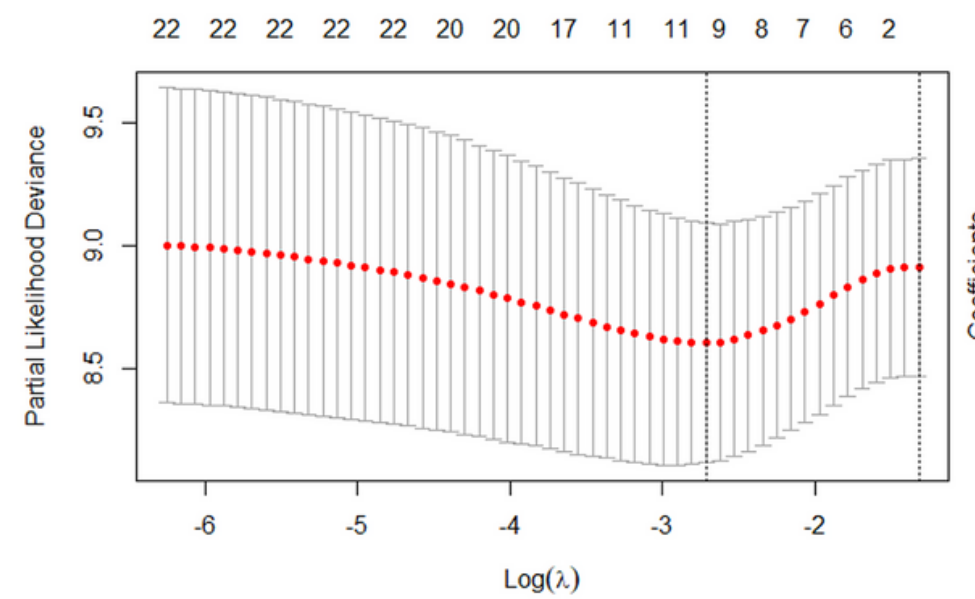

B

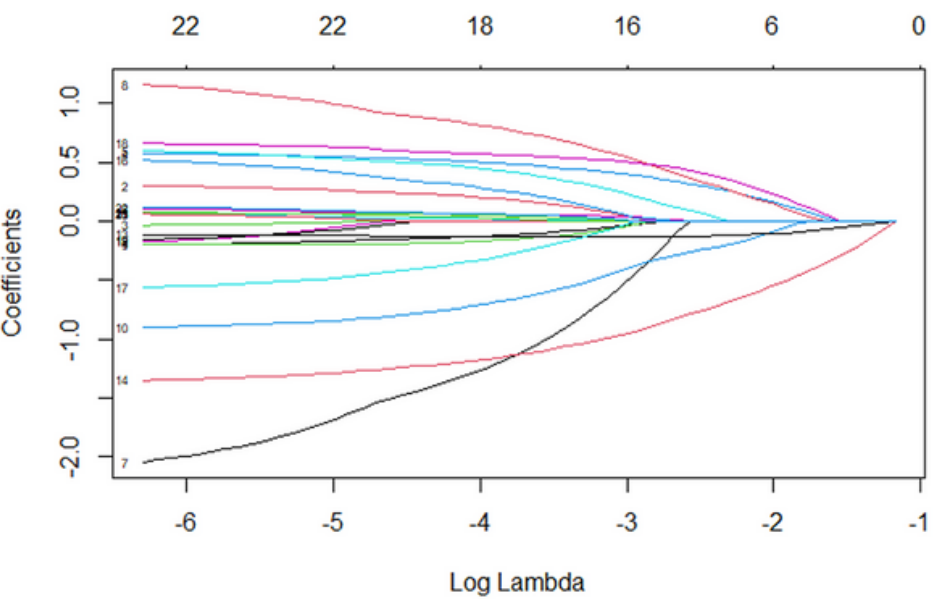

Figure 1

Demographic and clinicopathological characteristics screening applying the elastic net regression model for PFS. Final predictors include FIGO stage, Bulky tumor, Way of EBRT, Chemotherapy, C.C.D, ICBT, PLT pre-treatment, PLT in early stages of the treatment, and NEU in early stages of the treatment. (A) Suitable parameter $(\lambda)$ selection in the elastic net model used 10 -fold cross-validation via minimum criteria. The partial likelihood deviance (binomial deviance) curve versus log $(\lambda)$ was plotted. 2 dotted vertical lines were drawn at the optimal values applying the minimum criteria and the one standard error of the minimum criteria (the 1-SE criteria). (B) Elastic net coefficient profiles of the variables. A coefficient profile plot was produced against the $\log (\lambda)$ sequence.

A

$\begin{array}{llllllllllllllll}22 & 21 & 20 & 20 & 19 & 18 & 18 & 17 & 16 & 12 & 9 & 8 & 6 & 6 & 3 & 1\end{array}$

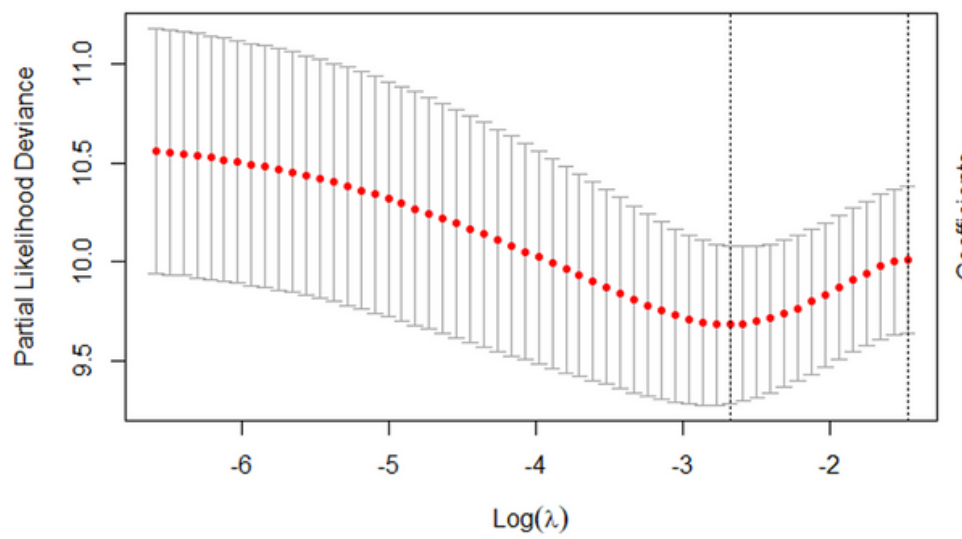

B

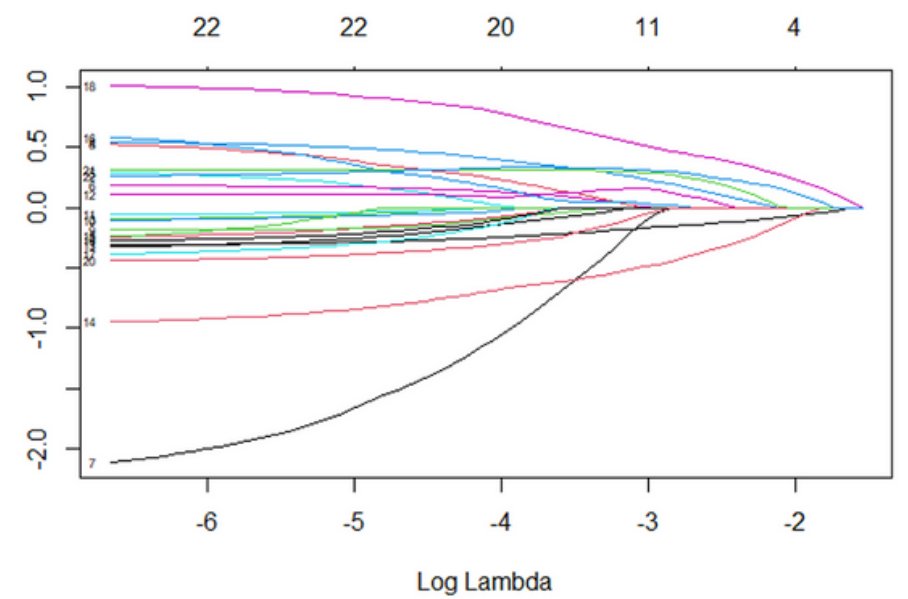

Figure 2 
Demographic and clinicopathological characteristics screening applying the elastic net regression model for OS. Final predictors include Bulky tumors, Chemotherapy, C.C.D, ICBT, PLT pre-treatment, and NEU in early stages of the treatment. (A) Suitable parameter $(\lambda)$ selection in the elastic net model used 10-fold cross-validation via minimum criteria. The partial likelihood deviance (binomial deviance) curve versus $\log (\lambda)$ was plotted. 2 dotted vertical lines were drawn at the optimal values applying the minimum criteria and the one standard error of the minimum criteria (the 1-SE criteria). (B) Elastic net coefficient profiles of the variables. A coefficient profile plot was produced against the $\log (\lambda)$ sequence.
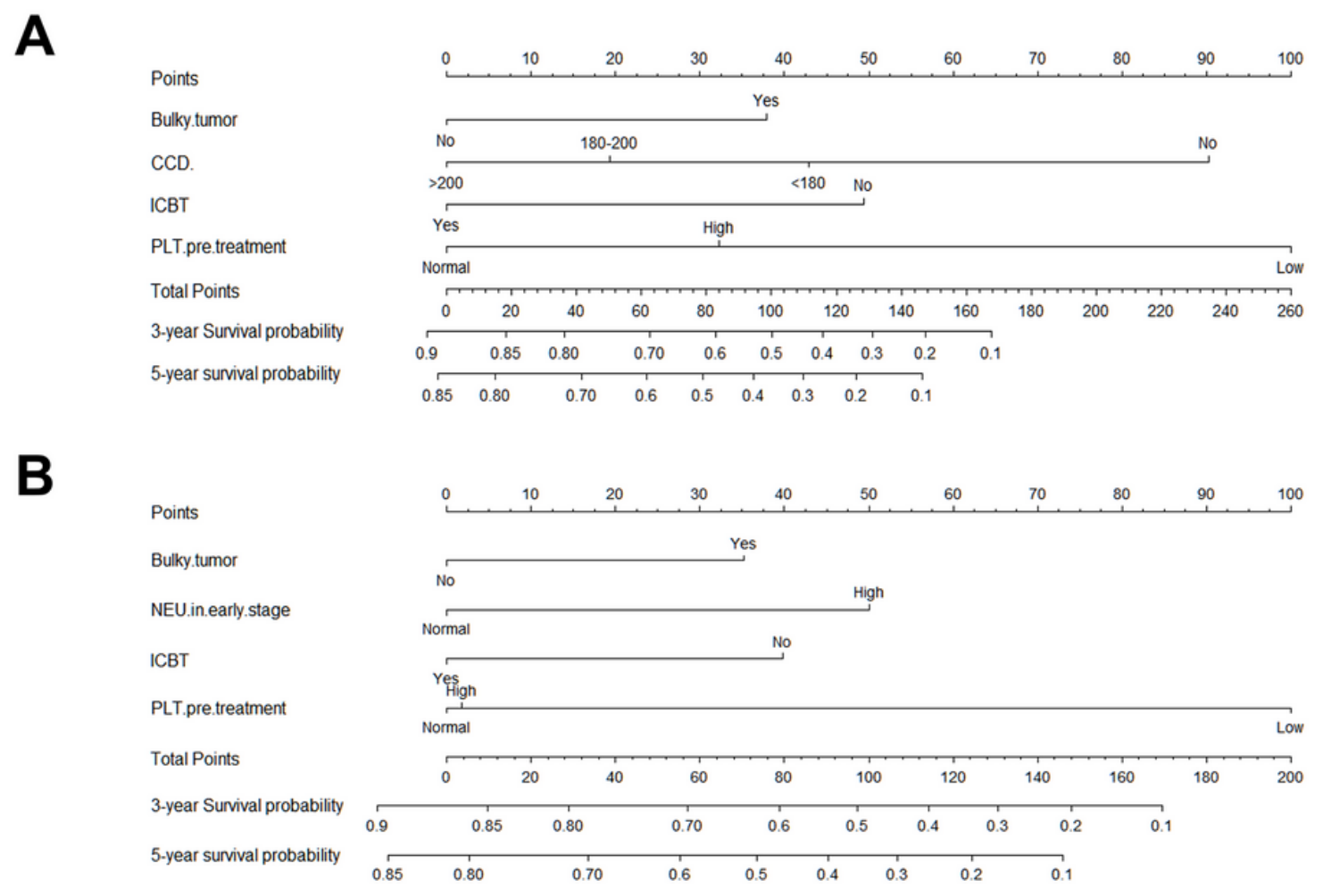

\section{Figure 3}

Nomogram for predicting the PFS and OS probability in LACC patients. (A) Nomogram to determine the likelihood of 3- or 5-year PFS. (B) nomogram to determine the likelihood of 3- or 5-year OS. 
A
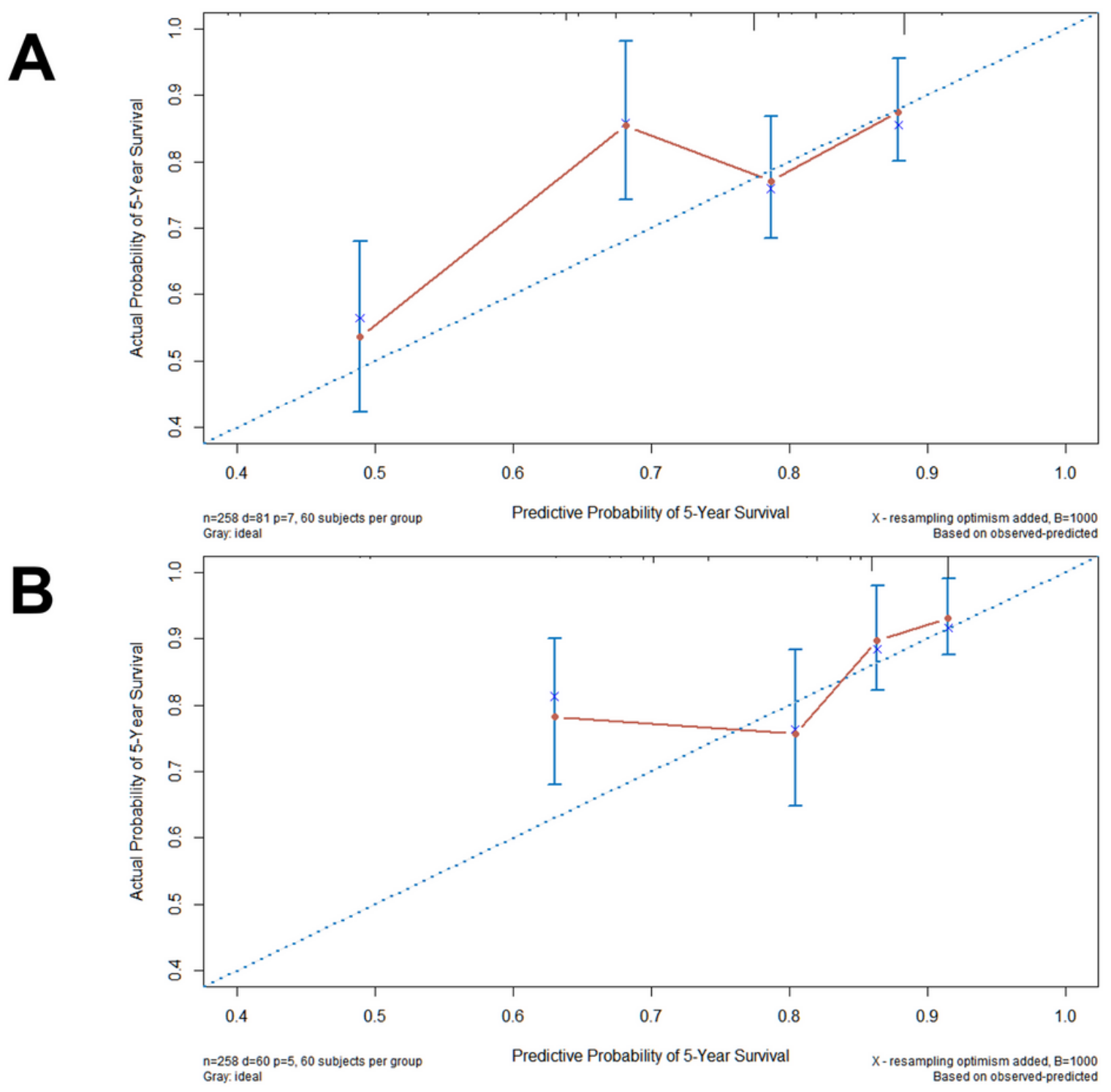

Figure 4

Calibration plots for 5-year PFS and 5-year OS with 95\% confidence interval of actual risk probability (yaxis), overpredicted risk probability by nomogram(x-axis). (A) Calibration plots for 5-year PFS. (B) Calibration plots for 5-year OS. 

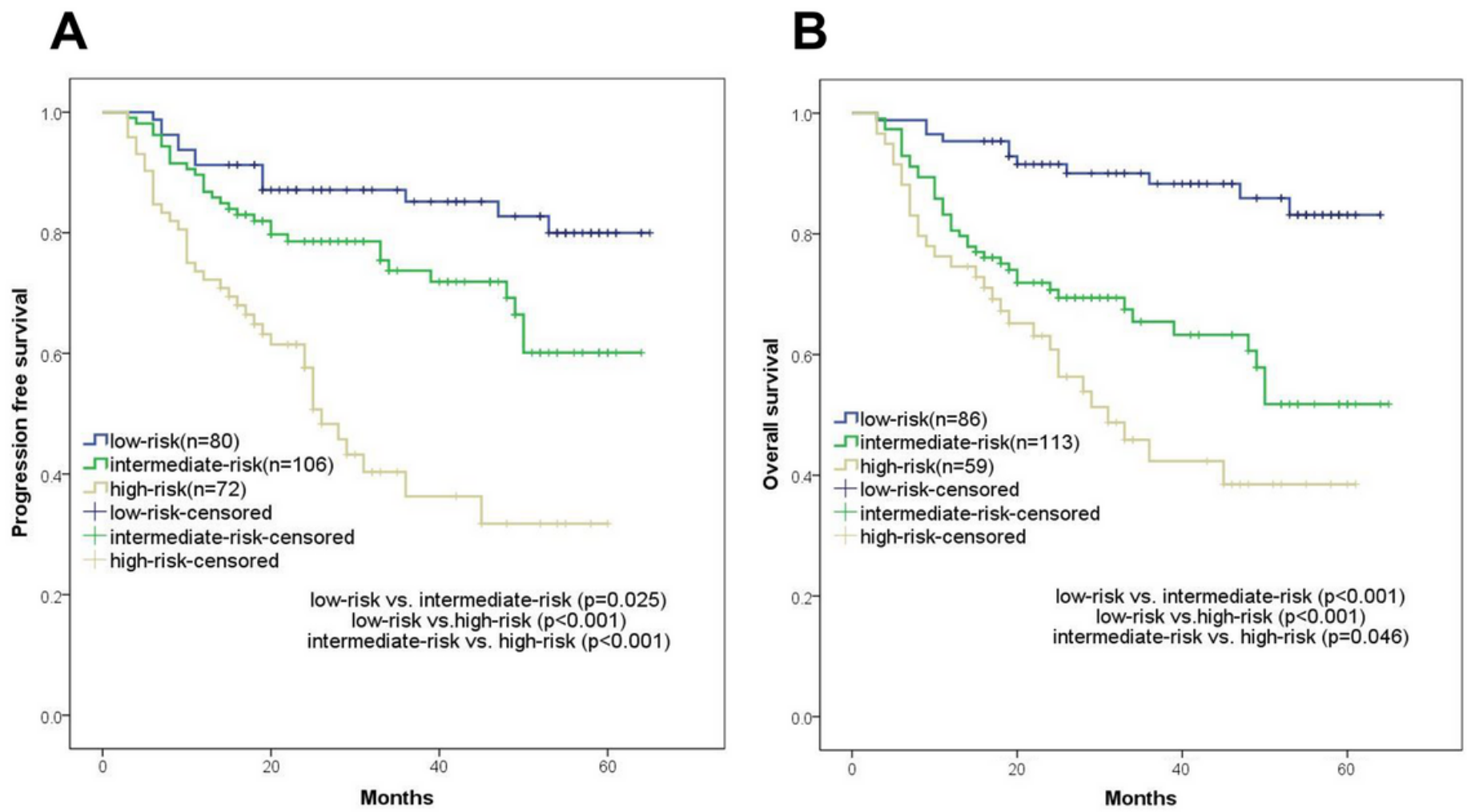

Figure 5

Kaplan-Meier curves of PFS and OS for patients in different groups of risk stratification. (A) PFS curve for patients in different groups $(p<0.05)$. (B) OS curve for patients in different groups $(p<0.05)$ 